\title{
Existence of Positive Periodic Solution of a Ratio-Dependent Predator- Prey System with Time Delays
}

\author{
Xinyi Chen
}

China Minorities Information Technology Institute, Northwest Minzu University, Lanzhou 730030, China

Keywords: predator-prey system; time delay; periodic solution; coincidence degree

Abstract. A periodic ratio-dependent predator-prey system with time delay is investigated. By using a continuation theorem based on coincidence degree theory, the sufficient conditions of the existence of periodic solution of the system are obtained, which generalizes the known result.

\section{Introduction}

Ratio-dependent predator-prey models have received much attention recently as more suitable ones for predator-prey interactions where predation involves searching process. A typical ratio-dependent predator-prey model can be express in the form

$$
\left\{\begin{array}{l}
y_{1}^{\prime}(t)=y_{1}(t)\left(a-b y_{1}(t)-\frac{c y_{2}(t)}{m y_{2}(t)+y_{1}(t)}\right], \\
y_{2}^{\prime}(t)=y_{2}(t)\left[-d+\frac{f y_{1}(t)}{m y_{2}(t)+y_{1}(t)}\right],
\end{array}\right.
$$

where $y_{1}$ and $y_{2}$ stand for prey and predator density, respectively. $a, b, c, d, f$ and $m$ are positive constants. For the ecological sense of system (1), we refer to [1] and reference therein. System (1) was systematically studied by Kuang and Beretta [1] and Arditi and coworkers[2-7]. They discussed global stability of the boundary equilibria, positive equilibrium, and permanence of the system. Therefore, paper [8] is interesting and important to study the following periodic ratio-dependent system with time delays

$$
\left\{\begin{array}{l}
y_{1}^{\prime}(t)=y_{1}(t)\left[a(t)-b(t) y_{1}\left(t-\tau_{1}\right)-\frac{c(t) y_{2}(t)}{m(t) y_{2}(t)+y_{1}(t)}\right], \\
y_{2}^{\prime}(t)=y_{2}(t)\left[-d(t)+\frac{f(t) y_{1}\left(t-\tau_{2}\right)}{m(t) y_{2}\left(t-\tau_{2}\right)+y_{1}\left(t-\tau_{2}\right)}\right]
\end{array}\right.
$$

with initial conditions

$$
y_{i}(s)=\varphi_{i}(s), s \in[-\tau, 0], \varphi_{i}(s)>0, \varphi_{i} \in C\left([-\tau, 0], R_{+}\right), i=1,2 .
$$

where $a(t), b(t), c(t), m(t), d(t)$ and $f(t)$ are strictly positive continuous $\omega$-periodic functions. $\tau_{1}$ and $\tau_{2}$ are nonnegative constants, $\tau=\max \left\{\tau_{1}, \tau_{2}\right\}$. They obtained the sufficient conditions of the positive periodic solution of the system as follows

Theorem1.1 Assume the following conditions are satisfied

$\left(\mathrm{H}_{1}\right) f(t)-d(t)>0$,

$\left(\mathrm{H}_{2}\right) \overline{m a-c}>0$.

Then system (2) has at least one positive $\omega$-periodic solution.

We will be concerned with a more general system as follows

$$
\left\{\begin{array}{l}
y_{1}^{\prime}(t)=y_{1}(t)\left[a(t)-b(t) y_{1}\left(t-\tau_{1}\right)-\frac{c(t) y_{2}^{p}(t)}{m(t) y_{2}^{p}(t)+y_{1}^{p}(t)}\right], \\
y_{2}^{\prime}(t)=y_{2}(t)\left[-d(t)+\frac{f(t) y_{1}^{p}\left(t-\tau_{2}\right)}{m(t) y_{2}^{p}\left(t-\tau_{2}\right)+y_{1}^{p}\left(t-\tau_{2}\right)}\right] .
\end{array}\right.
$$

initial conditions also is (3), where $a(t), b(t), c(t), m(t), d(t)$ and $f(t)$ are strictly positive continuous $\omega$-periodic functions. $p \geq 1$ is a real number. Our purpose in this paper is, by using the 
continuation theorem of coincidence degree theory, to establish the existence conditions of at least one positive $\omega$-periodic solution of system(4).

\section{Main results}

For convenience of use, we introduce the continuation theory [9] as follows.

Lemma2.1 Let $\Omega \subset X$ be an open bounded set. Let $L$ be a Fredholm mapping of index zero and $N$ be $L$-compact on $\bar{\Omega}$. Assume

(a) for each $\lambda \in(0,1), \quad x \in \partial \Omega \cap \operatorname{Dom} L, L x \neq \lambda N x$;

(b) for each $x \in \partial \Omega \cap \operatorname{KerL}, Q N x \neq 0$;

(c) $\operatorname{deg}\{J Q N, \Omega \cap \operatorname{Ker} L, 0\} \neq 0$.

Then $L x=N x$ has at least one solution in $\bar{\Omega} \cap \operatorname{Dom} L$.

Lemma2.2 $R_{+}^{2}=\left\{\left(y_{1}, y_{2}\right) \mid y_{i}>0, i=1,2\right\}$ is positive invariant set of system (4).

Proof From equation (4),we can obtain

$$
\begin{aligned}
& y_{1}(t)=y_{1}(0) \exp \left\{\int_{0}^{t}\left[a(s)-b(s) y_{1}\left(s-\tau_{1}\right)-\frac{c(s) y_{2}^{p}(s)}{m(s) y_{2}^{p}(s)+y_{1}^{p}(s)}\right] \mathrm{d} s\right\}>0, \text { for } y_{1}(0)>0, \\
& y_{2}(t)=y_{2}(0) \exp \left\{\int_{0}^{t}\left[-d(s)+\frac{f(s) y_{1}^{p}\left(s-\tau_{2}\right)}{m(s) y_{2}^{p}\left(s-\tau_{2}\right)+y_{1}^{p}\left(s-\tau_{2}\right)}\right] \mathrm{d} s\right\}>0, \text { for } y_{2}(0)>0 .
\end{aligned}
$$

The proof is complete.

In what follows we shall use the notations

$$
\bar{f}=\frac{1}{\omega} \int_{0}^{\omega} f(t) \mathrm{d} t, \quad f^{l}=\min _{t \in[0, \omega]} f(t), \quad f^{u}=\max _{t \in[0, \omega]} f(t)
$$

where $f$ is a continuous $\omega$-periodic function. Our main result in this paper is the following theorem about the existence of a positive $\omega$-periodic solution of system (4).

Theorem2.1 Assume the following conditions are satisfied

$\left(\mathrm{H}_{1}\right) f(t)-d(t)>0$,

$\left(\mathrm{H}_{2}\right) \overline{m a-c}>0$.

Then system (4) has at least one positive $\omega$-periodic solution.

Proof Let

$$
x_{1}(t)=\ln y_{1}(t), \quad x_{2}(t)=\ln y_{2}(t) .
$$

On substituting (5) into (4), we rewrite (4) in the form

$$
\begin{aligned}
& x_{1}^{\prime}(t)=a(t)-b(t) \exp \left(x_{1}\left(t-\tau_{1}\right)\right)-\frac{c(t)\left\{\exp \left(x_{2}(t)\right)\right\}^{p}}{m(t)\left\{\exp \left(x_{2}(t)\right)\right\}^{p}+\left\{\exp \left(x_{1}(t)\right)\right\}^{p}}, \\
& x_{2}^{\prime}(t)=-d(t)+\frac{f(t)\left\{\exp \left(x_{1}\left(t-\tau_{2}\right)\right)\right\}^{p}}{\left.m(t)\left\{\exp \left(x_{2}\left(t-\tau_{2}\right)\right)\right\}^{p}+\exp \left(x_{1}\left(t-\tau_{2}\right)\right)\right\}^{p}} .
\end{aligned}
$$

So to complete the proof, it suffices to show that system (6) has at least one $\omega$-periodic solution. Take

$$
\begin{gathered}
X=Y=\left\{\left(x_{1}(t), x_{2}(t)\right)^{T} \in C\left(\mathrm{R}, \mathrm{R}^{2}\right): x_{i}(t+\omega)=x_{i}(t), i=1,2\right\}, \\
\left\|\left(x_{1}(t), x_{2}(t)\right)^{T}\right\|=\max _{t \in[0, \omega]}\left(\left|x_{1}(t)\right|,\left|x_{2}(t)\right|\right) .
\end{gathered}
$$

Then $X$ and $Y$ are Banach spaces with the above norm $\|\cdot\|$, Set

$$
\begin{gathered}
L: \operatorname{Dom} L \subset X \rightarrow Y, \\
L\left(x_{1}(t), x_{2}(t)\right)^{T}=\left(x_{1}^{\prime}(t), x_{2}^{\prime}(t)\right)^{T},
\end{gathered}
$$

where $\operatorname{Dom} L=\left\{\left(x_{1}(t), x_{2}(t)\right)^{T} \in C^{1}\left(\mathrm{R}, \mathrm{R}^{2}\right)\right\}$, and $N: X \rightarrow Y$, 


$$
N\left[\begin{array}{l}
x_{1}(t) \\
x_{2}(t)
\end{array}\right]=\left[\begin{array}{l}
a(t)-b(t) \exp \left(x_{1}\left(t-\tau_{1}\right)\right)-\frac{c(t)\left\{\exp \left(x_{2}(t)\right)\right\}^{p}}{m(t)\left\{\exp \left(x_{2}(t)\right)\right\}^{p}+\left\{\exp \left(x_{1}(t)\right)\right\}^{p}} \\
-d(t)+\frac{f(t)\left\{\exp \left(x_{1}(t)\right)\right\}^{p}}{m(t)\left\{\exp \left(x_{2}(t)\right)\right\}^{p}+\left\{\exp \left(x_{1}(t)\right)\right\}^{p}}
\end{array}\right] .
$$

With these notations system (6) can be written in the form $L x=N x, x \in X$.

Obviously, $\operatorname{Ker} L=\mathrm{R}^{2}, \operatorname{Im} L=\left\{\left(x_{1}(t), x_{2}(t)\right)^{T} \in X: \int_{0}^{\omega} x_{i}(t) \mathrm{d} t=0, i=1,2\right\}$ is closed in $Y$, and $\operatorname{dim} \operatorname{Ker} L=\operatorname{codim} \operatorname{Im} L=2$. Therefore $L$ is a Fredholm mapping of index zero. Now define two projectors $P: X \rightarrow X$, and $Q: Y \rightarrow Y$ as

$$
P\left[\begin{array}{l}
x_{1}(t) \\
x_{2}(t)
\end{array}\right]=Q\left[\begin{array}{l}
x_{1}(t) \\
x_{2}(t)
\end{array}\right]=\left[\begin{array}{l}
\bar{x}_{1} \\
\bar{x}_{2}
\end{array}\right],\left[\begin{array}{l}
x_{1}(t) \\
x_{2}(t)
\end{array}\right] \in X=Y .
$$

Then $P$ and $Q$ are continuous projectors such that $\operatorname{Im} P=\operatorname{Ker} L, \operatorname{Ker} Q=\operatorname{Im} L=\operatorname{Im}(I-Q)$. We select $J$, the isomorphism of $\operatorname{Im} Q$ onto Ker $L$ as identity map. Furthermore through an easy computation we find that the inverse $K_{P}$ of $L_{P}$ has the form

$$
\begin{gathered}
K_{P}: \operatorname{Im} L \rightarrow \operatorname{Dom} L \cap \operatorname{Ker} P, \\
K_{P}(y)=\int_{0}^{t} y(s) d s-\frac{1}{\omega} \int_{0}^{\omega} \mathrm{d} t \int_{0}^{t} y(s) \mathrm{d} s .
\end{gathered}
$$

Then $Q N: X \rightarrow Y$ and $K_{P}(I-Q) N: X \rightarrow X$ read

$$
\begin{gathered}
Q N x=\left[\begin{array}{l}
\frac{1}{\omega} \int_{0}^{\omega}\left[a(t)-b(t) \exp \left(x_{1}\left(t-\tau_{1}\right)\right)-\frac{c(t)\left\{\exp \left(x_{2}(t)\right)\right\}^{p}}{m(t)\left\{\exp \left(x_{2}(t)\right\}^{p}+\left\{\exp \left(x_{1}(t)\right)\right\}^{p}\right.}\right] \mathrm{d} t \\
\frac{1}{\omega} \int_{0}^{\omega}\left[-d(t)+\frac{f(t)\left\{\exp \left(x_{1}\left(t-\tau_{2}\right)\right)\right\}^{p}}{m(t)\left\{\exp \left(x_{2}\left(t-\tau_{2}\right)\right)\right\}^{p}+\left\{\exp \left(x_{1}\left(t-\tau_{2}\right)\right)\right\}^{p}}\right] \mathrm{d} t
\end{array}\right], \\
K_{P}(I-Q) N x=\int_{0}^{t} N x(s) d s-\frac{1}{\omega} \int_{0}^{\omega} \mathrm{d} t \int_{0}^{t} N x(s) \mathrm{d} s-\left(\frac{1}{\omega}-\frac{1}{2}\right) \int_{0}^{\omega} N x(s) \mathrm{d} s .
\end{gathered}
$$

Clearly, $Q N$ and $K_{P}(I-Q) N$ are continuous. By using the Arzela-Ascoli theorem, it is not difficult to prove that $\overline{K_{P}(I-Q) N(\bar{\Omega})}$ is compact for any open bounded set $\Omega \subset X$. Moreover, $Q N(\bar{\Omega})$ is bounded. Therefore $N$ is $L$-compact on $\bar{\Omega}$ with any open bounded set $\Omega \subset X$. Corresponding to the operator equation $L x=\lambda N x, \lambda \in(0,1)$, we have

$$
\begin{aligned}
& x_{1}^{\prime}(t)=\lambda\left[a(t)-b(t) \exp \left(x_{1}\left(t-\tau_{1}\right)\right)-\frac{c(t)\left\{\exp \left(x_{2}(t)\right)\right\}^{p}}{m(t)\left\{\exp \left(x_{2}(t)\right)\right\}^{p}+\left\{\exp \left(x_{1}(t)\right)\right\}^{p}}\right], \\
& x_{2}^{\prime}(t)=\lambda\left[-d(t)+\frac{f(t)\left\{\exp \left(x_{1}\left(t-\tau_{2}\right)\right)\right\}^{p}}{\left.m(t)\left\{\exp \left(x_{2}\left(t-\tau_{2}\right)\right)\right\}^{p}+\exp \left(x_{1}\left(t-\tau_{2}\right)\right)\right\}^{p}}\right] .
\end{aligned}
$$

Suppose that $\left(x_{1}(t), x_{2}(t)\right)^{T} \in X$ is a solution of (7) for a certain $\lambda \in(0,1)$. Integrating (7) over the interval $[0, \omega]$, we obtain

$$
\begin{aligned}
& \int_{0}^{\omega} \lambda\left[a(t)-b(t) \exp \left(x_{1}\left(t-\tau_{1}\right)\right)-\frac{c(t)\left\{\exp \left(x_{2}(t)\right)\right\}^{p}}{m(t)\left\{\exp \left(x_{2}(t)\right)\right\}^{p}+\left\{\exp \left(x_{1}(t)\right)\right\}^{p}}\right] \mathrm{d} t=\int_{0}^{\omega} x_{1}^{\prime}(t) \mathrm{d} t=0, \\
& \int_{0}^{\omega} \lambda\left[-d(t)+\frac{f(t)\left\{\exp \left(x_{1}\left(t-\tau_{2}\right)\right)\right\}^{p}}{\left.m(t)\left\{\exp \left(x_{2}\left(t-\tau_{2}\right)\right)\right\}^{p}+\exp \left(x_{1}\left(t-\tau_{2}\right)\right)\right\}^{p}}\right] \mathrm{d} t=\int_{0}^{\omega} x_{2}^{\prime}(t) \mathrm{d} t=0 .
\end{aligned}
$$

That is

$$
\begin{aligned}
& \int_{0}^{\omega}\left[b(t) \exp \left(x_{1}\left(t-\tau_{1}\right)\right)+\frac{c(t)\left\{\exp \left(x_{2}(t)\right)\right\}^{p}}{m(t)\left\{\exp \left(x_{2}(t)\right)\right\}^{p}+\left\{\exp \left(x_{1}(t)\right)\right\}^{p}}\right] \mathrm{d} t=\int_{0}^{\omega} a(t) \mathrm{d} t=\bar{a} \omega \\
& \int_{0}^{\omega}\left[\frac{f(t)\left\{\exp \left(x_{1}\left(t-\tau_{2}\right)\right)\right\}^{p}}{\left.m(t)\left\{\exp \left(x_{2}\left(t-\tau_{2}\right)\right)\right\}^{p}+\exp \left(x_{1}\left(t-\tau_{2}\right)\right)\right\}^{p}}\right] \mathrm{d} t=\int_{0}^{\omega} d(t) \mathrm{d} t=\bar{d} \omega .
\end{aligned}
$$


It follows from (7) and (8) that

$$
\begin{aligned}
\int_{0}^{\omega}\left|x_{1}^{\prime}(t)\right| \mathrm{d} t & \leq \int_{0}^{\omega}\left[a(t)+b(t) \exp \left(x_{1}\left(t-\tau_{1}\right)\right)+\frac{\left.\left.c(t)\{\exp ) x_{2}(t)\right)\right\}^{p}}{m(t)\left\{\exp \left(x_{2}(t)\right)\right\}^{p}+\left\{\exp \left(x_{1}(t)\right)\right\}^{p}}\right] \mathrm{d} t \\
= & 2 \bar{a} \omega, \\
\int_{0}^{\omega}\left|x_{2}^{\prime}(t)\right| \mathrm{d} t & \leq \int_{0}^{\omega}\left[d(t)+\frac{f(t)\left\{\exp \left(x_{1}\left(t-\tau_{2}\right)\right)\right\}^{p}}{m(t)\left\{\exp \left(x_{2}\left(t-\tau_{2}\right)\right)\right\}^{p}+\left\{\exp \left(x_{1}\left(t-\tau_{2}\right)\right)\right\}^{p}}\right] \mathrm{d} t \\
& =2 \bar{d} \omega .
\end{aligned}
$$

Since $\left(x_{1}(t), x_{2}(t)\right)^{T} \in X$, there exist $\xi_{i}, \eta_{i} \in[0, \omega]$ such that

$$
\begin{aligned}
& x_{i}\left(\xi_{i}\right)=\min _{t \in[0, \omega]} x_{i}(t), \\
& x_{i}\left(\eta_{i}\right)=\max _{t \in[0, \omega]} x_{i}(t), \quad i=1,2 .
\end{aligned}
$$

Then from (8) we have

which implies

$$
\exp \left(x_{1}\left(\xi_{1}\right)\right) \int_{0}^{\omega} b(t) \mathrm{d} t \leq \int_{0}^{\omega} b(t) \exp \left(x_{1}\left(t-\tau_{1}\right)\right) \mathrm{d} t<\bar{a} \omega,
$$

$$
x_{1}\left(\xi_{1}\right)<\ln \frac{\bar{a}}{\bar{b}}:=A_{1} .
$$

This, together with (9), leads to

$$
x_{1}(t) \leq x_{1}\left(\xi_{1}\right)+\int_{0}^{\omega}\left|x^{\prime}(t)\right| d t<A_{1}+2 \bar{a} \omega .
$$

From (8), we also have

$$
\begin{aligned}
\exp \left(x_{1}\left(\eta_{1}\right)\right) \int_{0}^{\omega} b(t) \mathrm{d} t \geq \int_{0}^{\omega} b(t) \exp \left(x_{1}\left(t-\tau_{1}\right)\right) \mathrm{d} t \\
\quad=\bar{a} \omega-\int_{0}^{\omega} \frac{c(t)\left\{\exp \left(x_{2}(t)\right)\right\}^{p}}{m(t)\left\{\exp \left(x_{2}(t)\right)\right\}^{p}+\left\{\exp \left(x_{1}(t)\right)\right\}^{p}} \mathrm{~d} t \geq \frac{\overline{m a-c}}{m^{u}} \omega,
\end{aligned}
$$

which implies

$$
x_{1}\left(\eta_{1}\right)>\ln \frac{\overline{m a-c}}{\bar{b} m^{u}}:=A_{2} .
$$

This, together with (9), leads to

$$
x_{1}(t) \geq x_{1}\left(\eta_{1}\right)-\int_{0}^{\omega}\left|x^{\prime}(t)\right| d t>A_{2}-2 \bar{a} \omega
$$

It follows from (12) and (13) that

$$
\max _{t \in[0, \omega]}\left|x_{1}(t)\right|<\max \left\{\left|A_{1}\right|,\left|A_{2}\right|\right\}+2 \bar{a} \omega:=R_{1} .
$$

From (11) and the second equation of (7), we can derive

which is equivalent to

$$
-d\left(\xi_{2}\right)+\frac{f(t)\left\{\exp \left(x_{1}\left(\xi_{2}-\tau_{2}\right)\right)\right\}^{p}}{m\left(\xi_{2}\right)\left\{\exp \left(x_{2}\left(\xi_{2}-\tau_{2}\right)\right)\right\}^{p}+\left\{\exp \left(x_{1}\left(\xi_{2}-\tau_{2}\right)\right)\right\}^{p}}=0,
$$

$$
d\left(\xi_{2}\right) m\left(\xi_{2}\right)\left\{\exp \left(x_{2}\left(\xi_{2}-\tau_{2}\right)\right)\right\}^{p}=\left[f\left(\xi_{2}\right)-d\left(\xi_{2}\right)\right]\left\{\exp \left(x_{1}\left(\xi_{2}-\tau_{2}\right)\right)\right\}^{p} .
$$

Set $\xi_{2}-\tau_{2}=t_{0}+n \omega$, where $t_{0} \in[0, \omega], n$ is an integer. Since periodicity of $\left(x_{1}(t), x_{2}(t)\right)^{T}$, we get

$$
\begin{aligned}
& x_{1}\left(\xi_{2}-\tau_{2}\right)=x_{1}\left(t_{0}\right), \\
& x_{2}\left(\xi_{2}-\tau_{2}\right)=x_{2}\left(t_{0}\right) .
\end{aligned}
$$

It follows from (14), (15) and (16) that

$$
\left|x_{2}\left(t_{0}\right)\right| \leq \max \left\{\frac{1}{p}\left|\ln \left[\frac{f-d}{m d}\right]^{u}\right|, \frac{1}{p}\left|\ln \left[\frac{f-d}{m d}\right]^{l}\right|\right\}+R_{1}:=A_{3} .
$$

This, together with (10), leads to

$$
\max _{t \in[0, \omega]}\left|x_{2}(t)\right| \leq\left|x_{2}\left(t_{0}\right)\right|+\int_{0}^{\omega}\left|x_{2}^{\prime}(t)\right| d t<A_{3}+2 \bar{d} \omega:=R_{2} .
$$


On the other hand, let's consider algegraic equation

$$
\begin{aligned}
& \bar{a}-\bar{b} \exp \left(x_{1}\right)-\frac{\mu\left\{\exp \left(x_{2}\right)\right\}^{p}}{\omega} \int_{0}^{\omega} \frac{c(t)}{m(t)\left\{\exp \left(x_{2}\right)\right\}^{p}+\left\{\exp \left(x_{1}\right)\right\}^{p}} \mathrm{~d} t=0, \\
& -\bar{d}+\frac{\left\{\exp \left(x_{1}\right)\right\}^{p}}{\omega} \int_{0}^{\omega} \frac{f(t)}{m(t)\left\{\exp \left(x_{2}\right)\right\}^{p}+\left\{\exp \left(x_{1}\right)\right\}^{p}} d t=0,
\end{aligned}
$$

for $\left(x_{1}, x_{2}\right)^{T} \in \mathrm{R}^{2}$, where $\mu \in[0,1]$. Similar arguments in (14) and (17) show that

$$
\begin{gathered}
\left|x_{1}\right| \leq \max \left\{\left|A_{1}\right|,\left|A_{2}\right|\right\} \leq R_{1} \quad, \\
\left|x_{2}\right| \leq \max \left\{\frac{1}{p}\left|\ln \left[\frac{f-d}{m d}\right]^{u}\right|, \frac{1}{p}\left|\ln \left[\frac{f-d}{m d}\right]^{l}\right|\right\}+R_{1} \leq R_{2} .
\end{gathered}
$$

Clearly, $R_{1}$ and $R_{2}$ are independent of $\lambda$. Take $M=2 R_{1}+2 R_{2}$, and define

$$
\Omega=\left\{\left(x_{1}(t), x_{2}(t)\right)^{T} \in X:\left\|\left(x_{1}(t), x_{2}(t)\right)^{T}\right\|<M\right\} .
$$

It is clear that $\Omega$ verifies the conditions (a) and (b) in Lemma2.1.

In order to verify the condition (c) in Lemma2.1, we define

$$
\phi: \operatorname{Dom} L \bigcap \operatorname{Ker} L \times[0,1] \rightarrow X
$$

by

$$
\begin{aligned}
\phi\left(x_{1}, x_{2}, \mu\right) & =\left[\begin{array}{l}
\bar{a}-\bar{b} \exp \left(x_{1}\right) \\
-\bar{d}+\frac{\left\{\exp \left(x_{1}\right)\right\}^{p}}{\omega} \int_{0}^{\omega} \frac{f(t)}{m(t)\left\{\exp \left(x_{2}\right)\right\}^{p}+\left\{\exp \left(x_{1}\right)\right\}^{p}} \mathrm{~d} t
\end{array}\right] \\
& +\mu\left[\begin{array}{l}
\left.-\frac{\left\{\exp \left(x_{2}\right)\right\}^{p}}{\omega} \int_{0}^{\omega} \frac{c(t)}{m(t)\left\{\exp \left(x_{2}\right)\right\}^{p}+\left\{\exp \left(x_{1}\right)\right\}^{p}} \mathrm{~d} t\right]
\end{array}\right]
\end{aligned}
$$

where $\mu \in[0,1]$ is a parameter. If $\phi\left(x_{1}, x_{2}, \mu\right)=0$, then $\left(x_{1}, x_{2}\right)^{T}$ is the constant solution of the equation (18). From (19) and (20) we know

$$
\phi\left(x_{1}, x_{2}, \mu\right) \neq 0 \text { on } \partial \Omega \bigcap \operatorname{KerL} \text {. }
$$

So due to homotopy invariance theorem of topology degree we have

$$
\begin{aligned}
& \operatorname{deg}(\left.J Q N\left(x_{1}, x_{2}\right)^{T}, \Omega \cap \operatorname{Ker} L,(0,0)^{T}\right) \\
& \quad= \operatorname{deg}\left(\phi\left(x_{1}, x_{2}, 1\right), \Omega \cap \operatorname{Ker} L,(0,0)^{T}\right) \\
&= \operatorname{deg}\left(\phi\left(x_{1}, x_{2}, 0\right), \Omega \cap \operatorname{Ker} L,(0,0)^{T}\right) \\
&= \operatorname{deg}\left(\left(\bar{a}-\bar{b} \exp \left(x_{1}\right),-\bar{d}+\right.\right. \\
&\left.\left.\frac{\left\{\exp \left(x_{1}\right)\right\}^{p}}{\omega} \int_{0}^{\omega} \frac{f(t)}{m(t)\left\{\exp \left(x_{2}\right)\right\}^{p}+\left\{\exp \left(x_{1}\right)\right\}^{p}} d t\right)^{T}, \Omega \cap \operatorname{Ker} L,(0,0)^{T}\right) .
\end{aligned}
$$

It is easy to see that the following algebraic equation

$$
\begin{aligned}
& \bar{a}-\bar{b} \exp \left(x_{1}\right)=0, \\
& -\bar{d}+\frac{\left\{\exp \left(x_{1}\right)\right\}^{p}}{\omega} \int_{0}^{\omega} \frac{f(t)}{m(t)\left\{\exp \left(x_{2}\right)\right\}^{p}+\left\{\exp \left(x_{1}\right)\right\}^{p}} \mathrm{~d} t=0
\end{aligned}
$$

has a unique solution $\left(x_{1}^{*}, x_{2}^{*}\right)^{T} \in \mathrm{R}^{2}$. Thus

$$
\begin{aligned}
& \operatorname{deg}\left(\operatorname{JQN}\left(x_{1}, x_{2}\right)^{T}, \Omega \cap \operatorname{KerL},(0,0)^{T}\right) \\
& =\operatorname{sgn}\left[\bar{d}-\frac{\left\{\exp \left(2 x_{1}^{*}\right)\right\}^{p}}{\omega} \int_{0}^{\omega} \frac{-\bar{b} \exp \left(x_{1}^{*}\right)}{\left[m(t)\left\{\exp \left(x_{2}^{*}\right)\right\}^{p}+\left\{\exp \left(x_{1}^{*}\right)\right\}^{p}\right]^{2}}-\frac{\left\{\exp \left(x_{1}^{*}+x_{2}^{*}\right)\right\}^{p}}{\omega} \int_{0}^{\omega} \frac{m}{\left[m(t)\left\{\exp \left(x_{2}^{*}\right)\right\}^{p}+\left\{\exp \left(x_{1}^{*}\right)\right\}^{p}\right]^{2}}\right]=1 \neq 0 \text {. }
\end{aligned}
$$

By now we have proved that $\Omega$ satisfies all conditions in Lemma2.1. Hence (6) has at least one $\omega$-periodic solution. Accordingly, system (4) has at least one positive $\omega$-periodic solution. The proof is complete. 
Remark If $p=1$, the result becomes Theorem 1.1.

\section{Conclusion}

In this paper, we discussed the existence of positive periodic solution to a Ratio-Dependent Predator- Prey System with Time Delays. Sufficient conditions are obtained for the existence of positive periodic solution to system (4). Our work generalizes the known result.

\section{Acknowledgement}

This work supported by the open project program of Key Lab of China's National Linguistic Inf ormation Technology, Northwest Minzu University

\section{References}

[1] Kuang Y, Beretta E. Global qualitative analysis of a ratio-dependent predator-prey system[J]. J. Math. Biol, 1998, 36:389-406.

[2] Arditi R, Saiah H. Empirical evidence of the role of heterogeneity in ratio-dependent consumption[J]. ecology,1992,73(5):1544-1551.

[3] Arditi R, Gimzburg L R, Akcakaya H R. Variaiton in plankton densities among lakes: A case for ratio-dependent model[J]. American Naturalists, 1991,138(5):1287-1296.

[4] Arditi R, Perrin N, Saiah H. Functional response and heterogeneities: An experiment test with cladocerans[J]. OIKOS,1991,60(1):69-75.

[5] Chen F. On a periodic multi-species ecological model[J]. Applied Mathematics and Computation, 2005,171(1):492-510.

[6] Chen F. On a nonlinear non-autonomous predator-prey model with diffusion and distributed and distributed delay[J]. Journal of Computational and Applied Mathematics, 2005,180(1):33-49.

[7] Kuang Y. Delay differential equation with application in population dynamics[M]. NY: Academic Press,1993,67-70.

[8] Ding X, Cheng S. Existence of postitive periodic solution of a ratio-dependent predator-prey system with time delays[J]. Pure and Applied Mathematics,2006,22(1):112-116.

[9] Gaines R E, Mawhin J L. Coincidence Degree and Nonlinear Differential Equations[M]. Berlin: Springer-Verlag, 1977.

[10] Wang Y, Chaolu T, Chen Z. Using reproducing kernel for solving a class of singular weakly nonlinear boundary value problems[J].International Journal of Computer Mathematics, 2010,87(2):367-380.. 